\title{
Proteomic Analysis of Importin $\alpha$-interacting Proteins in Adult Mouse Brain
}

\author{
Masahiro Fukumoto ${ }^{1}$, Toshihiro Sekimoto ${ }^{2}$, and Yoshihiro Yoneda ${ }^{1,2,3^{*}}$ \\ ${ }^{1}$ Department of Frontier Biosciences, Osaka University Graduate School of Frontier Biosciences, Suita, \\ Osaka 565-0871, Japan, ${ }^{2}$ Department of Biochemistry, Osaka University Graduate School of Medicine, \\ Suita, Osaka 565-0871, Japan, ${ }^{3}$ CREST, Japan Science and Technology Agency, Osaka University, Suita, \\ Osaka 565-0871, Japan
}

\begin{abstract}
Many transport factors, such as importins and exportins, have been identified, and the molecular mechanisms underlying nucleocytoplasmic transport have been characterized. The specific molecules that are carried by each transport factor and the temporal profiles that characterize the movements of various proteins into or out of the nucleus, however, have yet to be elucidated. Here, we used a proteomic approach to identify molecules that are transported into the nuclei of adult mouse brain cells via importin $\alpha 5$. We identified 48 proteins in total, among which we chose seven to characterize more extensively: acidic (leucine-rich) nuclear phosphoprotein 32 family member A (Anp32a), far upstream element binding protein 1 (FUBP1), thyroid hormone receptor $\beta 1$ (TRß1), transaldolase 1, CDC42 effector protein 4 (CDC42-ep4), Coronin 1B, and brainspecific creatine kinase (CK-B). Analyses using green fluorescent protein (GFP)-fused proteins showed that Anp32a, FUBP1, and TRß1 were localized in the nucleus, whereas transaldolase 1, CDC42-ep4, CK-B, and Coronin 1B were distributed in both the cytoplasm and nucleus. Using a digitonin-permeabilized in vitro transport assay, we demonstrated that, with the exception of CK-B, these proteins were transported into the nucleus by importin $\alpha 5$ together with importin $\beta$ and Ran. Further, we found that leptomycin B (LMB) treatment increased nuclear CK-B-GFP signals, suggesting that CK-B enters the nucleus and is then exported in a CRM1dependent manner. Thus, we identified a comprehensive set of candidate proteins that are transported into the nucleus in a manner dependent on importin a5, which enhances our understanding of nucleocytoplasmic signaling in neural cells.
\end{abstract}

Key words: Nuclear protein transport/importin $\alpha /$ nuclear localization signal (NLS)/neural cells

\section{Introduction}

In eukaryotic cells, the nucleus is separated from the cytoplasm by a double membrane, the nuclear envelope. The nucleus contains most of the cell's genetic information and is the site of gene transcription and genome replication. This compartmentalization requires that karyophilic proteins, which are synthesized on free ribosomes in the cytoplasm, are imported into the nucleus, whereas mRNA is exported into the cytoplasm. This continuous traffic of macromolecules

\footnotetext{
*To whom correspondence should be addressed: Yoshihiro Yoneda, Department of Frontier Biosciences, Graduate School of Frontier Biosciences, Osaka University, 1-3 Yamada-oka, Suita, Osaka 565-0871, Japan.

Tel: +81-6-6879-4605, Fax: +81-6-6879-4609

E-mail: yyoneda@anat3.med.osaka-u.ac.jp

Abbreviations: MALDI, matrix-assisted laser desorption ionization; MS, mass spectrometry; NES, nuclear export signal; NLS, nuclear localization signal; NPC, nuclear pore complex; PMF, peptide mass fingerprint; PSD, postsynaptic density; TOF, time-of-flight.
}

between the nucleus and the cytoplasm occurs via NPCs, which penetrate the nuclear envelope (Davis, 1995).

The nuclear import and export of proteins are selectively mediated by specific amino-acid sequences termed nuclear localization signals (NLSs) and nuclear export signals (NESs), respectively. Classical basic-type NLSs, which have been extensively characterized, consist of one or two clusters of basic amino acids in the primary structure of a karyophilic protein (Pollard et al., 1996). The molecular mechanisms underlying nuclear protein import have been examined using typical NLS-containing import substrates. To determine and characterize factors required for the nuclear import of karyophiles, an in vitro digitonin-permeabilized semi-intact cell transport assay was developed (Adam et al., 1990) and used to create the following model. Nuclear import of cargo molecules containing typical NLSs starts with the formation of a stable complex in the cytoplasm. This NLS-dependent complex consists of two soluble 
transport factors: importin (karyopherin) $\alpha$ and $\beta$. Importin $\alpha$ recognizes the NLS and functions as an adaptor molecule to recruit importin $\beta$. Importin $\beta$ binds to NLS-cargo-bound importin $\alpha$ to form a ternary complex. Importin $\beta$ can also interact with components of the NPC (nucleoporins) and functions as a carrier molecule (Görlich et al., 1996).

The NLS-cargo/importin $\alpha /$ importin $\beta$ complex then translocates through a NPC via interactions between importin $\beta$ and nucleoporins. After translocation, a nuclear GTP-bound form of a small GTPase Ran (RanGTP) binds to importin $\beta$, thereby inducing a conformational change in importin $\beta$ and triggering dissociation of the complex. As a result, the cargo becomes free to function in the nucleus. RanGTP-bound importin $\beta$ then returns to the cytoplasm, as does importin $\alpha$, although importin $\alpha$ requires CAS in conjunction with RanGTP. Before the next round of transport, Ran bound to importin $\beta$ or CAS undergoes GTP hydrolysis in the cytoplasm to trigger dissociation of the export complex and recycling of the transport factors (for reviews, see Görlich and Mattaj, 1996; Mattaj and Englmeier, 1998; Yoneda, 2000; Stewart et al., 2001).

The fundamental principles of nuclear protein import have been elucidated using NLS-containing SV40 large T-antigen as a substrate. In addition, a variety of transport factors and pathways have been identified in various cell types. Although the budding yeast Saccharomyces cerevisiae contains a single gene encoding importin $\alpha$ (Srp1), importin $\alpha$ proteins are derived from a multigene family in mammals; the family can be divided into three distinct subgroups, which are differentially expressed in various tissues (Köhler et al., 1997, Tsuji et al., 1997). It has recently been shown that the expression of the importin $\alpha$ subgroups changes during neural differentiation and that these changes can trigger neural differentiation from mouse ES cells (Yasuhara et al., 2007). Interestingly, it has been reported that importin $\alpha$ alone can migrate into the nucleus in an importin $\beta /$ Ran-independent manner (Miyamoto et al., 2002) and that importin $\alpha$ transports CaMKIV (calcium/calmodulin-dependent protein kinase type IV) into the nucleus without utilizing importin $\beta$ (Kotera et al., 2005).

On the other hand, yeast contains 14 members of the importin $\beta$ family, and at least 22 importin $\beta$ family members have been identified in mammals. Importin $\beta$ family members usually function as either import or export receptors (for reviews, see Wozniak et al., 1998; Görlich and Kutay, 1999; Imamoto et al., 1995). In general, these proteins interact directly with their cargo. In fact, it has been demonstrated that importin $b$ itself mediates the nuclear import of such karyophilic proteins as the mature form of SREBP-2 (sterol regulatory element binding protein-2), Snail, Smad3, PTHrP (parathyroid hormone-related protein), and HIV Rev without the aid of adaptor molecules (Nagoshi et al., 1999; Lee et al., 2003; Kurisaki et al., 2001; Lam et al., 1999; Henderson and Percipalle, 1997).

Although cells may contain other unidentified transport factors, the sheer number of proteins that are transported between the nucleus and cytoplasm suggests that most transport factors recognize many distinct cargo molecules. Nevertheless, a systematic and comprehensive analysis of cargo molecules for each transport factor has yet to be performed. To address this issue, we have attempted to identify proteins that are transported by importin $\alpha 5$ in neural cells. We have focused on importin $\alpha 5$ and neural cells because importin $\alpha 5$ is highly expressed in neurons and is localized in dendrites as well as soma. Furthermore, we have recently shown that importin $\alpha 5$ plays important roles in neural differentiation and that importin $\alpha$ is involved in the nuclear import of CaMKIV (Yasuhara et al., 2007; Kotera et al., 2005), a major contributor to $\mathrm{Ca}^{2+}$-induced CREB (cAMP responsive element-binding protein)-mediated signaling in neurons (Enslen et al., 1994; Matthews et al., 1994). We also previously demonstrated that importin $\alpha 5$, but not importin $\alpha 1$, mediates the nuclear import of STAT1 (signal transducer and activator of transcription 1) (Sekimoto et al., 1997), suggesting that neural importin $\alpha 5$-binding proteins may include other signal transduction molecules. Finally, importin $\alpha$ constitutively associates with dynein in peripheral nerve axons, an important interaction for retrograde transport of molecules that signal nerve injury (Hanz et al., 2003). Little, however, is known about how nuclear protein transport is regulated in polarized cells, such as neurons.

In this study, we used a pull-down assay combined with mass spectrometry to identify 48 proteins from crude mouse brain extract that interact with importin $\alpha 5$ directly or indirectly. We also confirmed that some of the proteins migrated into the nucleus when transiently expressed in HeLa cells and/or primary neurons. Furthermore, using a digitonin-permeabilized semi-intact cell transport assay, we showed that some of the identified proteins were transported into the nucleus in an importin $\alpha 5 / \beta$-dependent manner.

\section{Experimental Procedures}

\section{Pull-down experiment}

Twenty adult (24 to 36 weeks old) C57BL/6 mice (SLC, Japan) were used for these experiments. Brains were removed quickly under ether anesthesia and immersed in a homogenizing buffer [10 mM HEPES (pH 7.3), 0.32 M sucrose, 0.1\% Triton X-100, $1 \mathrm{mM}$ EDTA, $5 \mathrm{mM}$ DTT, phosphatase inhibitors $(5 \mathrm{mM} \mathrm{NaF}$ and $1 \mathrm{mM}$ $\mathrm{Na}_{3} \mathrm{VO}_{4}$ ), $1 \mathrm{mM}$ p-(amidinophenyl)methanesulfonyl fluoride, and $1 \mu \mathrm{g} / \mathrm{ml}$ each leupeptin, pepstatin and aprotinin] at $4{ }^{\circ} \mathrm{C}$, followed by dissection of cerebral cortex. Tissues $(4.8 \mathrm{~g})$ were homogenized in $20 \mathrm{ml}$ of homogenizing buffer with a Teflon/Potter homogenizer using 20 gentle up and down strokes. The homogenate was then centrifuged at $100,000 \times \mathrm{g}$ for $1 \mathrm{~h}$. The supernatant was divided into aliquots and purified using recombinant glutathione S-transferase (GST)-bound glutathione-Sepharose 4B (GE Healthcare) filtration.

Purified GST-importin $\alpha 5$ (mouse, $12 \mathrm{mg}$ ) and GST (4 mg) were 
mixed in $10 \mathrm{ml}$ of phosphate buffered saline (PBS) containing $0.1 \%$ Triton $\mathrm{X}-100$ in the presence of glutathione-Sepharose $(1 \mathrm{ml})$ for $2 \mathrm{~h}$. After extensive washing, the glutathione-Sepharose beads were collected and incubated with $10 \mathrm{ml}$ of the homogenates for $30 \mathrm{~min}$. After the supernatants were removed, the glutathioneSepharose beads were washed five times with wash buffer $(20 \mathrm{mM}$ HEPES at pH 7.3, 5\% glycerol, 0.01\% Tween 20, 0.5 mM EGTA, 1 mM DTT, phosphatase inhibitors, and protease inhibitors), which was followed by the addition of $5 \mathrm{ml}$ of elution buffer $(20 \mathrm{mM}$ HEPES at pH 7.3, $2 \mathrm{M} \mathrm{NaCl}, 5 \%$ glycerol, $0.01 \%$ Tween $20,0.5$ $\mathrm{mM}$ EGTA, $1 \mathrm{mM}$ DTT, and protease inhibitors) and gentle rotation for $30 \mathrm{~min}$. Each 5-ml elution fraction was pooled, dialyzed overnight against dialysis buffer (20 mM HEPES at pH 7.3, 0.01\% Tween 20, $0.5 \mathrm{mM}$ EGTA, and $1 \mathrm{mM}$ DTT), and concentrated using a Vivaspin 6 (10 kDa molecular weight cut-off) concentrator (Vivascience). The samples were subjected to SDS-PAGE on $10 \%$ and $4-20 \%$ gradient acrylamide gels (Daiichi Pure Chemicals). For two-dimensional gel electrophoresis, each elution fraction was concentrated using the trichloroacetic acid/acetone precipitation method. Two-dimensional gel electrophoresis was carried out using an IPGphor II apparatus (GE Healthcare) according to the manufacturer's instructions.

\section{Plasmids and proteins}

cDNAs encoding full-length mouse Anp32a, transaldolase 1, TR $\beta 1$, FUBP1, CDC42-ep4, CK-B, or Coronin 1B were cloned from mouse brain total RNA using RT-PCRs, and verified by sequencing. All of the constructs expressed in transfectants were cloned into the pEGFP-N1 and pEGFP-N3 vectors (Clontech). Site-directed mutagenesis was performed using a QuikChange mutagenesis kit (Stratagene) with appropriate oligonucleotides. All coding regions of the constructs were sequenced to confirm the reading frames. To prepare GST-tagged proteins, cDNAs encoding full-length proteins were inserted in-frame into the pGEX-6P-1 and pGEX-6p-3 vectors (GE Healthcare), and introduced into the Escherichia coli BL21 (DE3) strain. Expression was induced by the addition of $0.1 \mathrm{mM}$ isopropyl- $\beta$-D-thiogalactopyranoside for $12 \mathrm{~h}$ at $18^{\circ} \mathrm{C}$ and GST fusion proteins were purified using glutathione-Sepharose according to the manufacturer's instructions. HA-importin $\beta$, Ran, p10/NTF2, and GST-importin $\alpha 5$ were expressed and purified as described previously (Imamoto et al., 1995; Sekimoto et al., 1997).

\section{HeLa cell cultures, transfection, and indirect immunofluorescence}

HeLa cells were maintained in Dulbecco's modified Eagle's Medium (Sigma) supplemented with 10\% fetal bovine serum and antibiotics at $37^{\circ} \mathrm{C}$ in a $10 \% \mathrm{CO}_{2}$ incubator. For the in vitro cell-free transport assay, HeLa cells were seeded at $1 \times 10^{6}$ cells $/ \mathrm{ml}$ on eight-well glass slides (ICN) for $24-48 \mathrm{~h}$ before the assay. HeLa cell transfection was carried out using Lipofectamine 2000 (Invitrogen) according to the manufacturer's protocol. Twelve hours after transfection, the cells were fixed with $3.7 \%$ formaldehyde, permeabilized with $0.5 \%$ Triton X-100, and blocked with $3 \%$ skim milk. The subcellular localization of GFP-fusion proteins was then detected using rabbit anti-GFP antibody (Molecular Probes), followed by incubation with Alexa Fluor 488 goat anti-rabbit IgG antibody (Molecular Probes). The samples were observed under a Zeiss Axiophot 2 fluorescence microscope with Plan-Neofluar objective lens (Carl Zeiss, 40×/0.75).

\section{Primary culture of neurons, transfection, and indirect immunofluorescence}

Hippocampus tissue was dissected from embryonic day 18 SpragueDawley rats and digested with $0.25 \%$ trypsin in Hanks' buffered salt solution. Neurons were suspended in neurobasal medium (Invitrogen) supplemented with B27 and $0.5 \mathrm{mM} \mathrm{L-glutamine} \mathrm{and}$ plated at $3.0 \times 10^{5}$ cells $/ 35-\mathrm{mm}$ plate on polyethyleneimine-coated tissue culture dishes at $37^{\circ} \mathrm{C}$ in $8 \% \mathrm{CO}_{2}$. On day 10 after plating, neurons were transfected with expression vectors using Trans Messenger (Qiagen) according to the manufacturer's protocol. Twenty-four hours after transfection, the neurons were fixed with 4\% paraformaldehyde in PBS for $20 \mathrm{~min}$ at room temperature and permeabilized with $0.5 \%$ Triton $\mathrm{X}-100$ in PBS for 10 min at room temperature. The cells were blocked with 3\% skim milk in PBS for $1 \mathrm{~h}$ at room temperature followed by incubation with the same solution containing rabbit anti-GFP antibody (Molecular Probes) and mouse monoclonal anti-MAP2 antibody (Sigma) for $1 \mathrm{~h}$ at room temperature. After washing three times with PBS, the cells were incubated with Alexa 488-labeled donkey anti-rabbit IgG and Alexa 568-labeled goat anti-mouse IgG (Molecular Probes) in blocking solution for $1 \mathrm{~h}$ at room temperature. The samples were then washed three times with PBS and counterstained with $1 \mathrm{mg} /$ $\mathrm{ml}$ Hoechst 33342 . Confocal images were obtained at $512 \times 512$ pixels using a LSM510 laser-scanning microscope with a PlanApochromat lens (Carl Zeiss, 63×/1.4).

\section{Cell-free transport assay}

An in vitro cell-free transport assay was performed essentially as described previously (Adam et al., 1990), with the following modifications. Digitonin-permeabilized HeLa cells were incubated at $4^{\circ} \mathrm{C}$ for $10 \mathrm{~min}$ and washed twice with ice-cold transport buffer $(20$ $\mathrm{mM}$ HEPES at $\mathrm{pH} 7.3,110 \mathrm{mM}$ potassium acetate, $5 \mathrm{mM}$ sodium acetate, $2 \mathrm{mM}$ magnesium acetate, $0.5 \mathrm{mM}$ EGTA, $1 \mathrm{mM}$ DTT, and $1 \mu \mathrm{g} / \mathrm{ml}$ each aprotinin, pepstatin, and leupeptin) to minimize the amount of residual protein in the cytoplasm. The cells were incubated at $30^{\circ} \mathrm{C}$ for $20 \mathrm{~min}$ with transport substrates/transport factors and an ATP regeneration system (1 mM ATP, 20 units/ml creatine phosphokinase, and $5 \mathrm{mM}$ creatine phosphate) in a total volume of $10 \mu \mathrm{l}$ per sample. GST-fused Anp32a, transaldolase 1, TR $\beta 1$, FUBP1, CDC42-ep4, CK-B, and Coronin 1B (10 pmol each) were used as transport substrates. After incubation, the cells were fixed with $4 \%$ paraformaldehyde in transport buffer, followed by permeabilization of the nuclear membrane using $0.5 \%$ Triton X-100 in PBS and immunostaining of GST using primary anti-GST monoclonal antibody (Molecular Probes) and secondary 
Alexa Fluor 488 goat anti-mouse IgG antibody (Santa Cruz Biotechnology). The nuclear import mixture containing GSTNLS-GFP (10 pmol) consisted of importin $\alpha 5$ (5 pmol), HAimportin $\beta$ (5 pmol), RanGDP (10 pmol), p10/NTF2 (10 pmol), and an ATP regeneration system adjusted with transport buffer. Ehrlich ascites tumor cell extract was prepared essentially as described previously (Imamoto et al., 1995) except that the concentration of the extract was adjusted using an ultrafiltration device (Vivascience).

\section{Solution-binding assay and immunoblotting}

Purified GST fusion proteins (200 pmol each), and GST (200 pmol) or importin a5 $(200 \mathrm{pmol})$ were mixed in $1 \mathrm{ml}$ of transport buffer containing $0.05 \%$ Triton X-100 in the presence of glutathione-Sepharose at $4^{\circ} \mathrm{C}$ for $3 \mathrm{~h}$. After extensive washing, bound proteins were eluted with SDS sample buffer, subjected to $10 \%$ SDS-PAGE, transferred to a nitrocellulose membrane, and Western blotted. After the membrane was blocked with 5\% skim milk in PBS, it was incubated with primary goat anti-karyopherin a1 (importin a5; Santa Cruz Biotechnology) polyclonal antibody for $2 \mathrm{~h}$ at room temperature and then with secondary horseradish peroxide (HRP)-conjugated anti-goat IgG antibody (Pierce). After washing the membrane with PBS containing $0.05 \%$ Tween 20 , the protein bands were visualized with an enhanced chemiluminescence detection kit (GE Healthcare). Purified GST fusion proteins were stained with Coomassie brilliant blue (CBB) solution.

\section{Identification of proteins using matrix-assisted laser desorption ionization (MALDI) mass spectrometry}

One- and two-dimensional gels were stained with silver stain according to the manufacturer's protocol (Shevchenko et al., 1996). Selected bands and spots from the gel were excised, destained in destaining buffer (20 mM EDTA, $25 \mathrm{mM}$ Tris at $\mathrm{pH} 8.0$, and $30 \%$ acetonitrile), washed with $60 \%$ acetonitrile, and dried in a SpeedVac (Savant). Then, the samples were reduced with $20 \mathrm{mM}$ DTT at $56^{\circ} \mathrm{C}$ for $30 \mathrm{~min}$ and alkylated with $55 \mathrm{mM}$ iodoacetamide in $25 \mathrm{mM}$ ammonium bicarbonate at $37^{\circ} \mathrm{C}$ for $30 \mathrm{~min}$ in the dark (Sechi and Chait, 1998). The processed gels were treated with trypsin (Promega) at $37^{\circ} \mathrm{C}$ for at least $6 \mathrm{~h}$. Tryptic peptides were extracted twice with $30 \mu \mathrm{l}$ of extraction solution containing $60 \%$ acetonitrile and $0.1 \%$ trifluoroacetic acid in water. The combined extracts were concentrated to $10 \mu \mathrm{l}$ and cleaned up with ZipTip C18 pipette tips (Millipore) according to the manufacturer's instructions. The peptides were eluted from the ZipTip with $0.5 \mu \mathrm{l}$ of extraction solution, and were spotted on a MALDI target plate with an $\alpha$-cyano-4-hydroxycinnamic acid matrix using the drieddroplet method.

MALDI-TOF MS spectra of tryptic digests were obtained using a Voyager DE-RP mass spectrometer (PerSeptive Biosystems) under the control of Voyager Biospectrometry Workstation. MS spectra were recorded in positive ion reflector mode in a mass range from $600-3500 \mathrm{Da}$, and with an ion acceleration voltage of $20 \mathrm{kV}$. GRAMS/386 (PerSeptive Biosystems) was used for spectra analyses and peak selection. All MS spectra were internally calibrated using autoproteolytic trypsin fragments and externally calibrated using angiotensin I (Novabiochem). Peak data, excluding trypsin autolytic peptides and other known background peaks, were entered in the online database search MASCOT version 2.1.01 (Matrix Science) for peptide mass fingerprint (PMF) comparisons with the NCBI nonredundant databases (NCBInr 20091212; 10157076 sequences for the final search) using permutations of the following search parameters: missed trypsin cleavages (0-2) and MS tolerance in daltons $(0.2,0.4)$. Fixed and variable modifications were considered (Cys as an S-carbamidomethyl derivate and Met as oxidized methionine), and the taxonomy was defined as Mus musculus. Only proteins with a statistically significant MASCOT identity score $(P<0.05)$ were accepted, and monoisotopic peaks were used.

\section{Results}

\section{Identification and classification of importin a5-interacting proteins in brain cells}

To identify molecules that interact with importin $\alpha 5$ in neural cells, we first prepared total cell extracts from adult mouse brain. We then pulled down binding partners using recombinant GST-fused importin $\alpha 5$ and separated the proteins using a combination of SDS-PAGE and twodimensional gel electrophoresis (Fig. 1 and data not shown). Approximately 100 bands were detected in SDS-PAGE, approximately 80 of which were identified as single bands. To identify the proteins, all of the 80 single bands were in-gel digested and analyzed with the aid of MALDI-TOF MS instruments. Mascot database searches using PMF spectra were also performed. The remaining 32 protein bands were not identified in the database, which can be attributed largely to the low frequency of these bands. Furthermore, approximately 50 spots were selected from 2D-gel and the 16 proteins were identified. However, all of the 16 proteins were included in the protein list identified from SDS-PAGE gel. Therefore, all the MS spectra for protein identification, which are listed in Supplementary Table I, were obtained from SDS-PAGE. As a result, 48 proteins were identified, and are listed in Supplementary Table I with the protein name, NCBI accession number, theoretical molecular weight and $\mathrm{p} I$ values, number of peptide masses, sequence coverage, MASCOT score, and known biological functions.

Based on the NCBI database, proteins identified in this study were classified into several functional groups: transport factors, components of metabolic pathways, receptors, proteins with GTPase activity, cytoskeleton components, transcription factors, chaperones, RNA-binding proteins, signal transducers, and other proteins (Fig. 2A). Their primary localization profiles are registered in the database as "nucleus," "nucleus and cytoplasm," "cytoplasm," or "membrane" (Fig. 2B). 


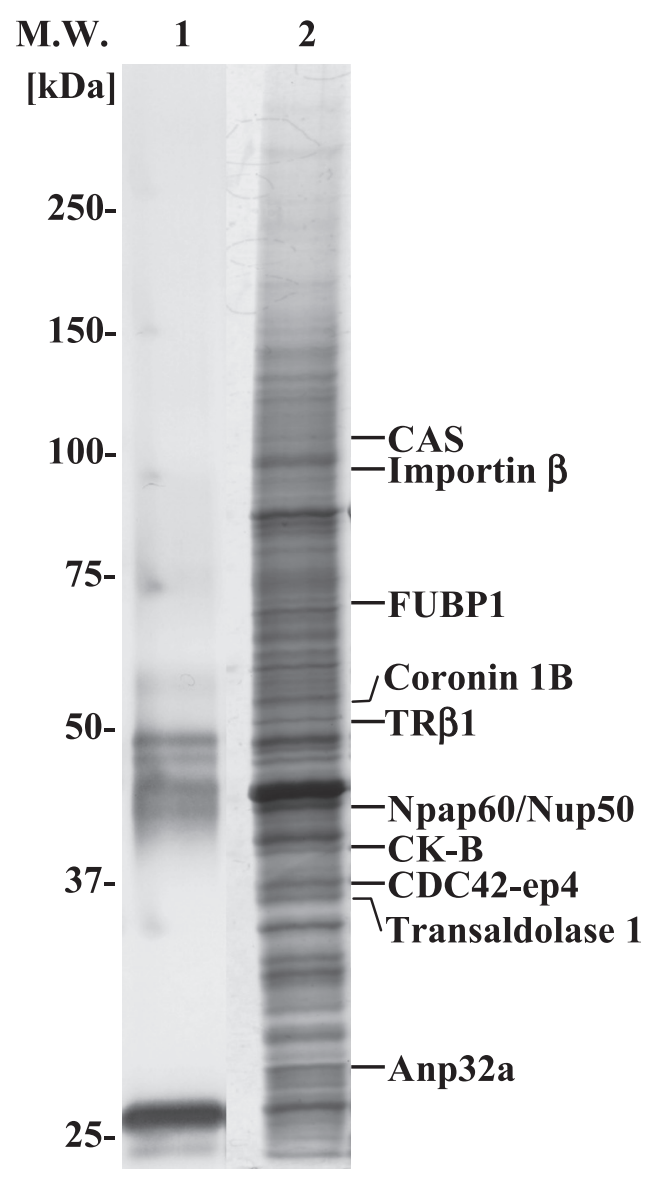

Fig. 1. A silver-stained SDS-PAGE gel (10\% acrylamide). Immobilized importin $\alpha 5$ was used to bind potential transport cargoes from mouse brain extract. Eluted proteins were separated using SDS-PAGE and detected with silver staining. Individual protein bands were isolated and identified using MALDI-TOF mass spectrometry. Eluted fraction from GST beads (lane 1) and from GST-importin $\alpha 5$ beads (lane 2). The position of transport factors and seven proteins analyzed in this study are indicated on the right.

\section{In vitro binding of the identified proteins with importin 25}

To gain a better understanding of the function of importin $\alpha 5$ in nuclear-cytoplasmic transport, it is important to investigate how diverse proteins are actually transported by importin $\alpha 5$. To this end seven proteins, whose transport mechanisms had not yet been reported, were selected for more detailed analysis from groups with multiple functions: transcription factors (Anp32a, TR $\beta 1$, and FUBP1), cytoskeletal proteins (CDC42-ep4 and Coronin 1B), and components of metabolic pathways (transaldolase 1 and CK-B).

To determine whether these proteins interact with importin $\alpha 5$ directly, recombinant full-length importin $\alpha 5$ was incubated with bacterially expressed recombinant GST-fused proteins that were immobilized on glutathione-Sepharose. After extensive washing, bound importin $\alpha 5$ was eluted with SDS sample buffer, analyzed using SDS-PAGE, and detected by immunoblotting with anti-importin $\alpha 5$ antibody. As shown in Fig. 3, the seven recombinant proteins directly interacted with importin $\alpha 5$. FUBP1 bound to importin $\alpha 5$ most strongly, whereas CDC42-ep4 showed relatively weak binding to importin $\alpha 5$. The results suggest that these proteins are candidate cargo molecules of importin $\alpha 5$.

\section{Intracellular localizations of the candidate cargo proteins}

As described above, the proteins identified in this study may not all localize in the nucleus. For instance, Anp32a, FUBP1, and TR $\beta 1$ have been previously classified as nuclear, whereas transaldolase 1, CDC42-ep4, Coronin 1B, and CK-B are reported to localize in the cytoplasm. Therefore, to examine whether the seven proteins migrate into the nucleus, we cloned cDNAs encoding the proteins into a mammalian expression vector and expressed them transiently in HeLa cells and primary hippocampal neurons.

Because proteins smaller than $\sim 40 \mathrm{kDa}$ have been reported to enter the nucleus through the NPC via passive diffusion (Görlich, 1998), we constructed full-length fusion proteins tagged with GFP at their carboxyl termini. As shown in Fig. 4 and Fig. 5, Anp32a, FUBP1, and TR $\beta 1$ strongly accumulated in the nuclei of both HeLa cells and primary neurons, which is consistent with the published literature.

On the other hand, transaldolase 1 and CDC42-ep4 were distributed not only in the cytoplasm but also in the nucleus. CK-B showed strong signals in the cytoplasm, but weak signals in the nucleus, which is consistent with a previous report showing that CK-B expression was detected in nuclear matrix fractions extracted from human colorectal tumors (Balasubramani et al., 2006). In addition, we found that Coronin 1B was mainly distributed in the cytoplasm, yet a significant amount of the protein was localized in the nuclei of primary neurons (Fig. 5). Although these four proteins have been thought to localize and function only in the cytoplasm, our findings suggest that they may continuously shuttle between the nucleus and the cytoplasm.

To test this possibility, we expressed the GFP-tagged proteins in HeLa cells and incubated the cells with LMB, a specific inhibitor of CRM1, which functions as an export receptor for leucine-rich NES-containing proteins (Nishi et al., 1994). We found that LMB treatment increased nuclear signals from CDC42-ep4-GFP, transaldolase 1-GFP, and CK-B-GFP, indicating that these proteins shuttle between the nucleus and the cytoplasm and that their export is mediated by CRM1. In contrast, LMB treatment did not affect the subcellular localization of Coronin $1 \mathrm{~B}$, suggesting that Coronin 1B may be exported from the nucleus in a CRM1independent manner (Fig. 6). 
A
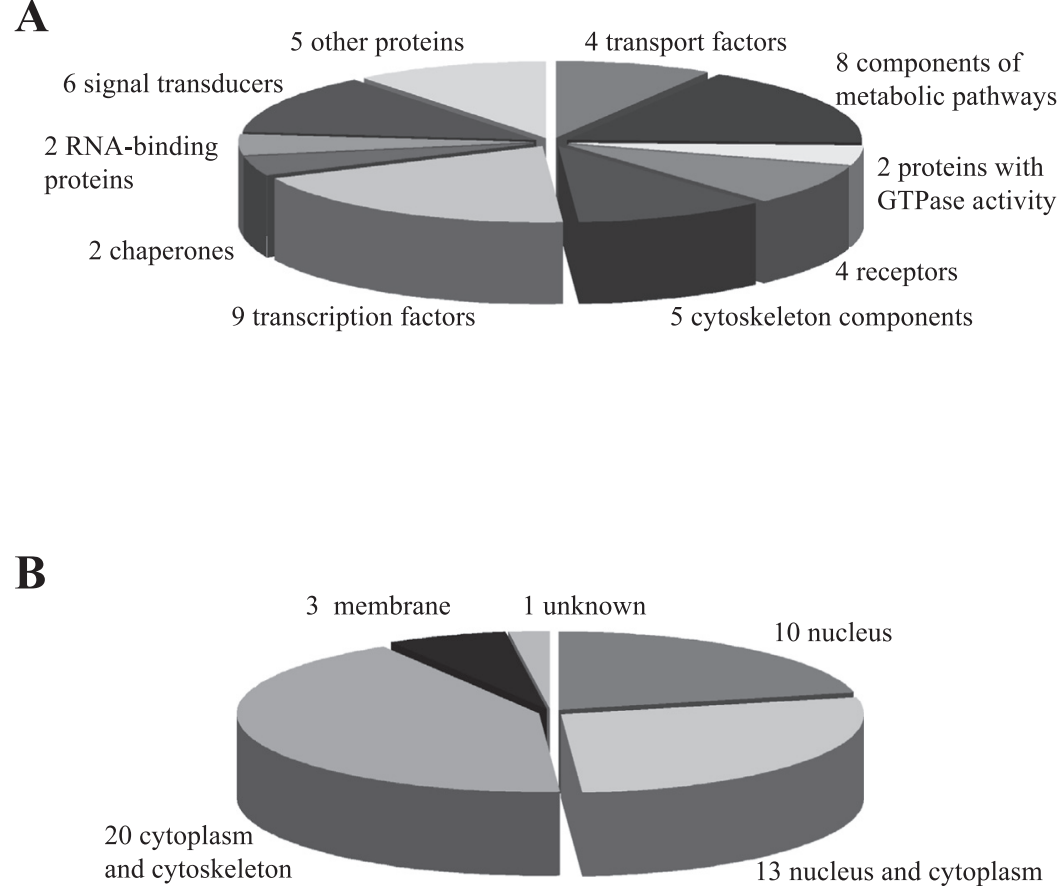

Fig. 2. Classification of importin $\alpha 5$-interacting proteins identified from mouse brain. A, Biological function. Transport proteins (8.3\%), components of metabolic pathways (22.9\%), receptors (8.3\%), proteins with GTPase activity (4.2\%), cytoskeleton components (8.3\%), transcription factors (18.8\%), RNAbinding proteins $(4.2 \%)$, chaperones $(4.2 \%)$, signal transducers (10.4\%), and others (10.4\%) are shown. B, Subcellular localization. Proteins that localized in the nucleus $(20.8 \%)$, nucleus and cytoplasm $(27.1 \%)$, cytoplasm $(45.8 \%)$, and membrane $(6.3 \%)$ are shown.

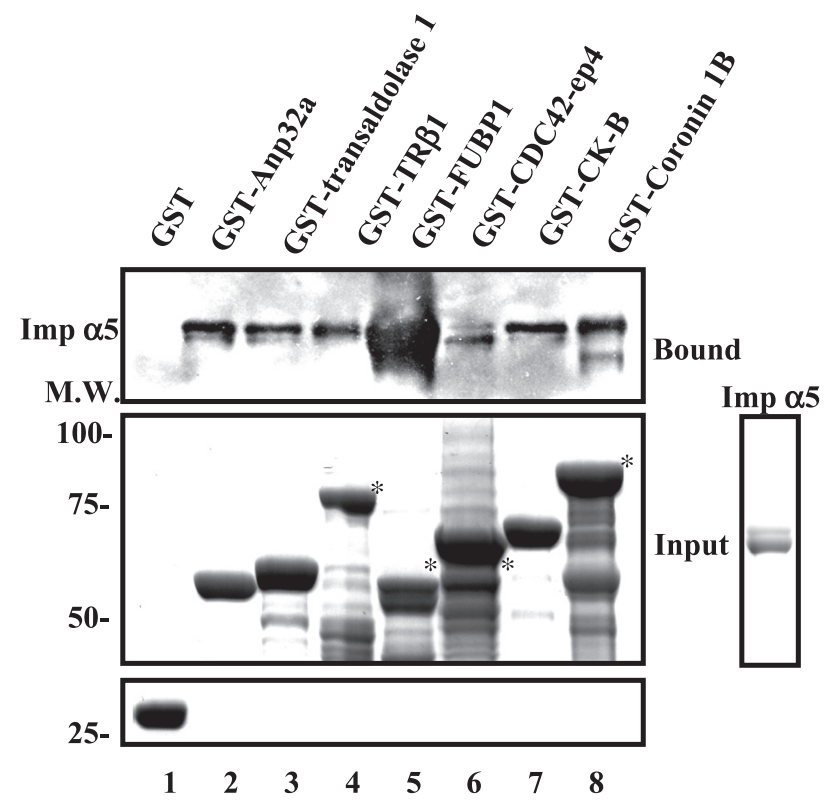

Fig. 3. Interactions between importin $\alpha 5$ and seven identified proteins GST fusion proteins were immobilized on glutathione-Sepharose 4B. After extensive washing, bound proteins were eluted with SDS sample buffer and analyzed on immunoblots using anti-karyopherin $\alpha 1$ (importin $\alpha 5$ ) antibody. As a control experiment, GST alone was used (lane 1). Input GST fusion proteins were separated using SDS-PAGE and stained with CBB solution. Asterisk (*) indicates the positions of full-length GST fusion proteins.

\section{In vitro assay for importin a5-mediated nuclear import of the identified proteins}

To confirm whether the seven proteins are transported into the nucleus via importin $\alpha 5 / \beta$ in conjunction with Ran, we performed an in vitro transport assay using digitoninpermeabilized semi-intact HeLa cells. Consistent with the transient expression experiments, recombinant GST-Anp32a, GST-TR $\beta 1$, and GST-FUBP1 were efficiently transported into nuclei via an importin $\alpha 5 / \beta$-mediated pathway (Fig. 7). We also found that recombinant GST-transaldolase 1, GSTCDC42-ep4, and GST-Coronin 1B strongly accumulated in nuclei in the presence of importin $\alpha 5 / \beta$, Ran, and a crude cytosolic extract of Ehrlich ascites tumor cells. Our results indicate that these proteins, which were thought to function in the cytoplasm, can enter the nucleus in an importin $\alpha 5 / \beta$ dependent manner.

In contrast, we did not observe nuclear accumulation of recombinant GST-CK-B under our in vitro assay conditions, demonstrating that $\mathrm{CK}-\mathrm{B}$ is not transported into the nucleus in an importin $\alpha 5 / \beta$-dependent manner. As described above, however, a weak CK-B-GFP signal was observed in the nuclei of cultured cells, and LMB treatment increased nuclear CK-B-GFP signals, indicating that CK-B enters the nucleus and is then exported from the nucleus in a CRM1dependent manner. Collectively, these results suggest that transaldolase 1, CDC42-ep4, CK-B, and Coronin 1B are 

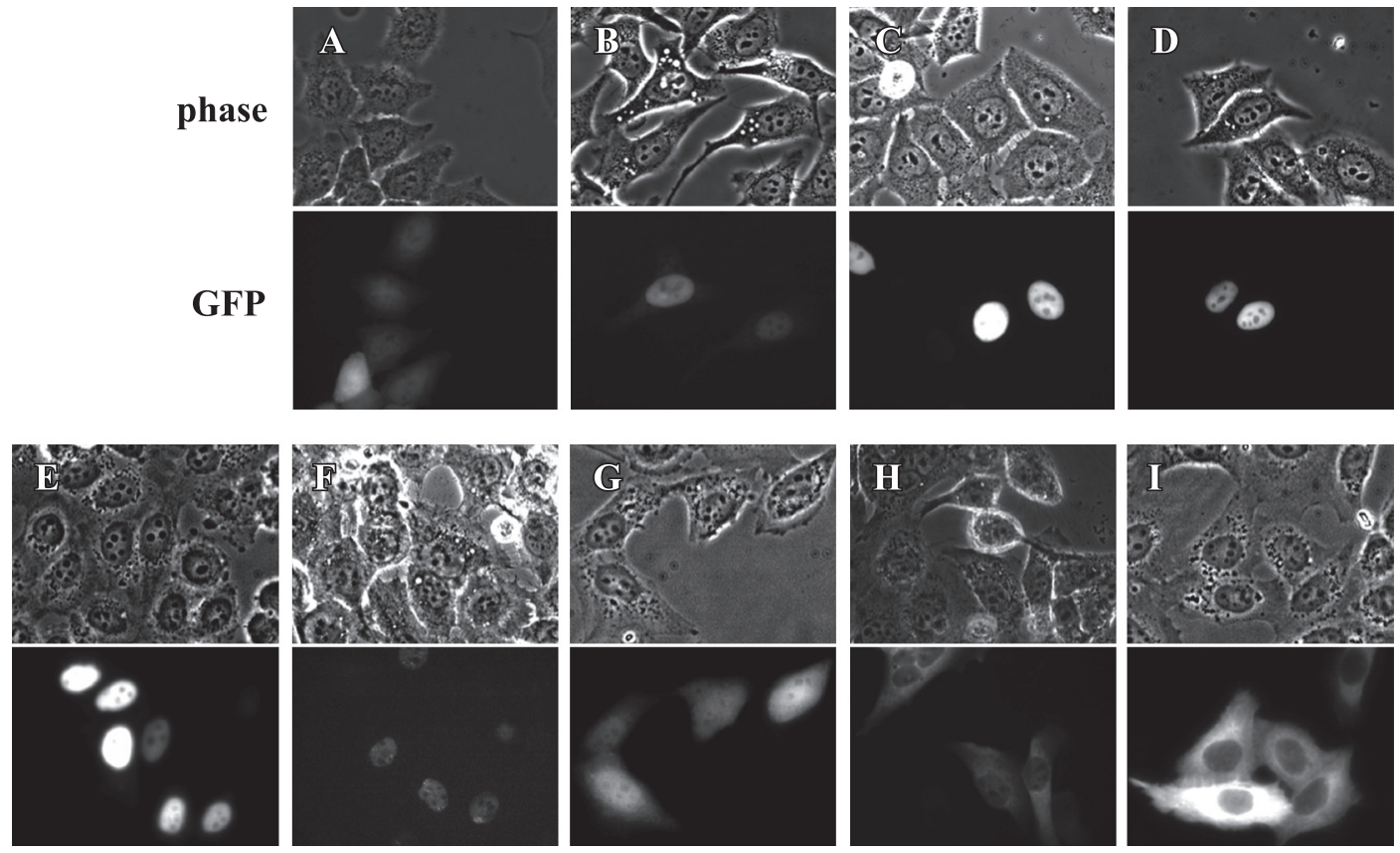

Fig. 4. Subcellular localization of importin $\alpha 5$-binding proteins tagged with GFP in HeLa cells. A, GFP alone. B, Importin $\alpha 5$. C, Anp32a. D, Transaldolase 1. E, TR $\beta 1$. F, FUBP1. G, CDC42-ep4. H, CK-B. I, Coronin 1B. GFP-tagged proteins were observed under a Zeiss Axiophot 2 fluorescence microscope.

good candidates for signaling molecules in neural cells, although it is possible that importin $\alpha 5$ may interact with CK-B in ways other than as a transport factor.

\section{Discussion}

To gain a better understanding of nucleocytoplasmic transport, it is important to determine which molecules are transported by each transport factor. Here, we have attempted to identify a comprehensive set of cargo molecules that are transported into nuclei by importin $\alpha 5 / \beta$ using adult mouse brain extracts. We identified 48 importin $\alpha 5$-interacting proteins which can be classified into a number of functional groups, including intracellular transport factors, components of metabolic pathways, transcription factors, RNA-binding proteins, and cytoskeletal proteins. Of note, the previously reported intracellular localization profiles of these proteins were not only nuclear, but also cytoplasmic, or nuclear and cytoplasmic.

As expected, the identified set contained proteins that are known to function in the nucleus, such as transcriptionrelated factors (thyroid hormone receptor corepressor, TR $\beta 1$, GATA6, and thyroid transcription factor 1), RNA-binding proteins (putative pre-mRNA splicing factor and heterogeneous nuclear ribonucleoprotein K), FUBP1, Anp32a, MAP kinase 1, and STAT5a. Indeed, Anp32a, a subunit of the INHAT (inhibitor of acetyltransferases) complex (Seo et $a l ., 2002)$, was efficiently transported into the nucleus by importin $\alpha 5 / \beta$ in our in vitro transport assay. Furthermore, we showed that nuclear import of TRb1 and FUBP1 is mediated by importin $\alpha 5$. TR $\beta 1$ is a nuclear hormone receptor that regulates transcription by binding to thyroid hormone response elements in target genes (Baumann et al., 2001). FUBP1 is a single-stranded DNA-binding protein that functions as a regulator of c-Myc expression and as an ATP-dependent DNA helicase (Duncan et al., 1994). Our results indicate that these factors are mediated into the nucleus by importin $\alpha 5 / \beta$ and may play an important role in the development of the neural cells.

The identified proteins also included a variety of cytoskeleton-related proteins, such as MAP2a, Coronin 1B, tubulin $\alpha 2, \gamma$-actin, $\beta$-actin, and CDC42-ep4. To examine the functional roles of the interactions between importin $\alpha 5$ and these cytoskeleton-related proteins, we focused on Coronin 1B and CDC42-ep4. Interestingly, we found that Coronin $1 \mathrm{~B}$ and $\mathrm{CDC} 42-\mathrm{ep} 4$ can enter the nuclei through an importin $\alpha 5 / \beta$-mediated pathway, suggesting that these cytoskeleton-related proteins shuttle between the nucleus and cytoplasm. Coronin 1B is localized at the leading edge of migrating fibroblasts (Cai et al., 2007), and was also identified as an important factor for neurite outgrowth in a neuronal regeneration model (Di et al., 2005). CDC42-ep4 is thought to be a cytoplasmic protein involved in several cellular processes and Rho protein signal transduction (Joberty et al., 1999). It has been recently reported that 

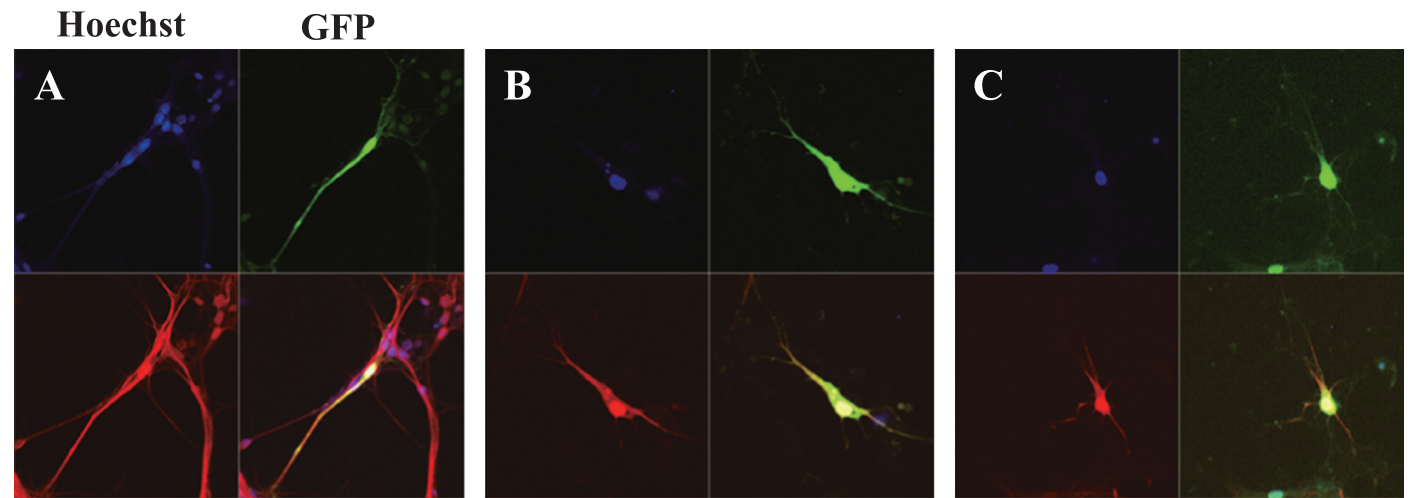

\section{Map-2 Marge}
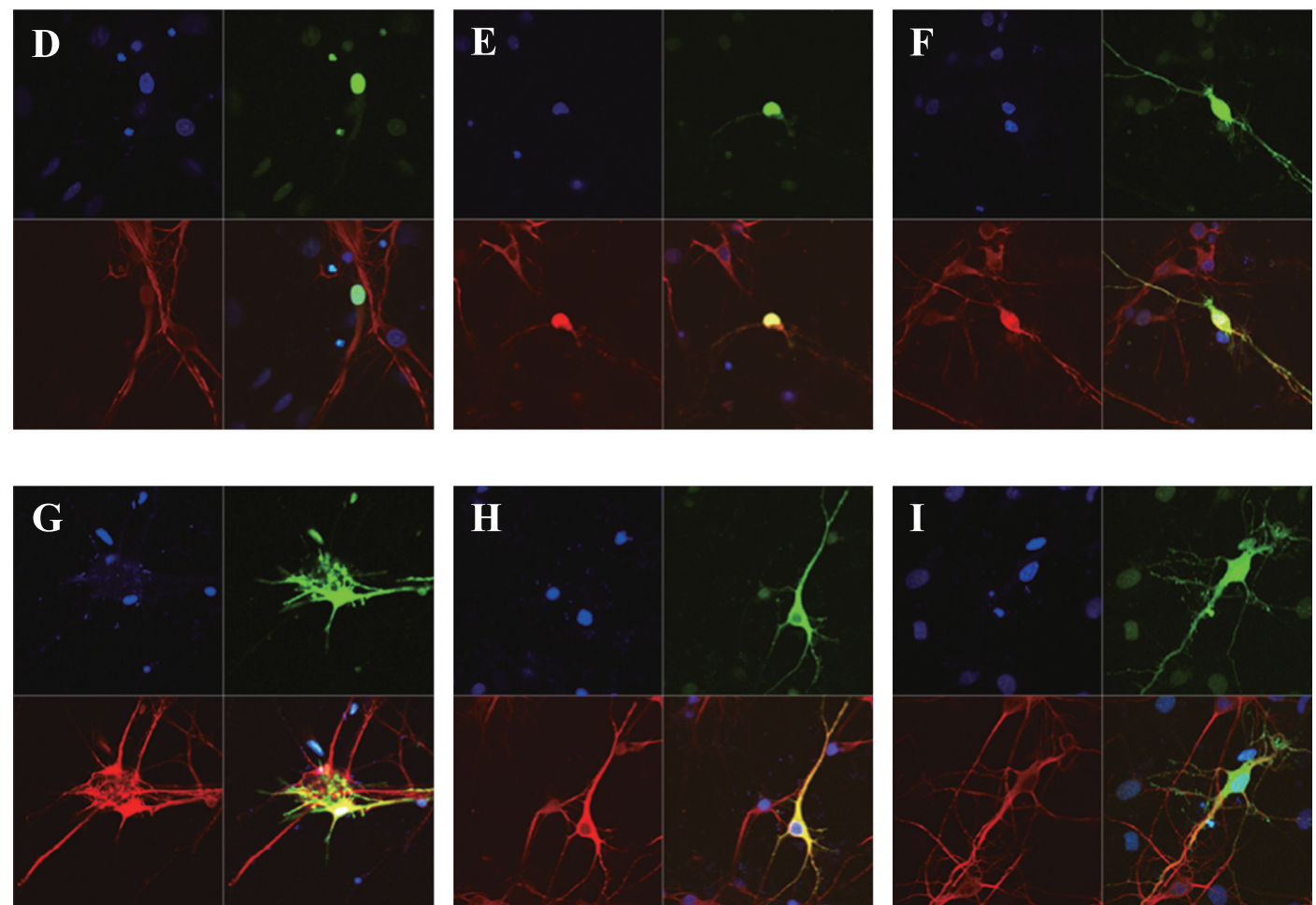

Fig. 5. Subcellular localization of importin $\alpha$ 5-binding proteins tagged with GFP in primary cultured hippocampal neurons. A, GFP alone. B, Importin a5. C, Anp32a. D, Transaldolase 1. E, TRß1. F, FUBP1. G, CDC42-ep4. H, CK-B. I, Coronin 1B. Nuclei were stained with Hoechst 33342. Images of paraformaldehyde-fixed cells were taken using an LSM510 confocal system.

mDia2, an effector of Rho, shuttles between the nucleus and cytoplasm through an importin $\alpha / \beta$ - and CRM1-mediated nuclear transport mechanism (Miki et al., 2009). These results suggest that some cytoskeletal proteins that predominantly localize in the cytoplasm may play roles in the nucleus.

In addition, we found that importin $\alpha 5$ interacts with some enzymes, transaldolase 1 and CK-B, which were thought to localize and function in the cytoplasm. Transaldolase 1 is part of the pentose phosphate pathway, which produces ribose-5-phosphate for nucleic acid synthesis and regulates
NADPH levels for lipid biosynthesis. Transiently expressed transaldolase 1 was predominantly localized in the nuclei of HeLa cells and primary hippocampal neurons, and recombinant protein was efficiently transported into nuclei with importin $\alpha 5 / \beta$ in an in vitro transport assay. It is likely that this protein functions in the nucleus, although we have not determined whether the aforementioned metabolic pathway occurs in the nucleus. The ATP-generating enzyme CK-B, mainly expressed in brain tissue, is regarded as a cytoplasmic protein. However, several reports have shown nuclear localization for CK-B (Chen et al., 1995). Although it was not 


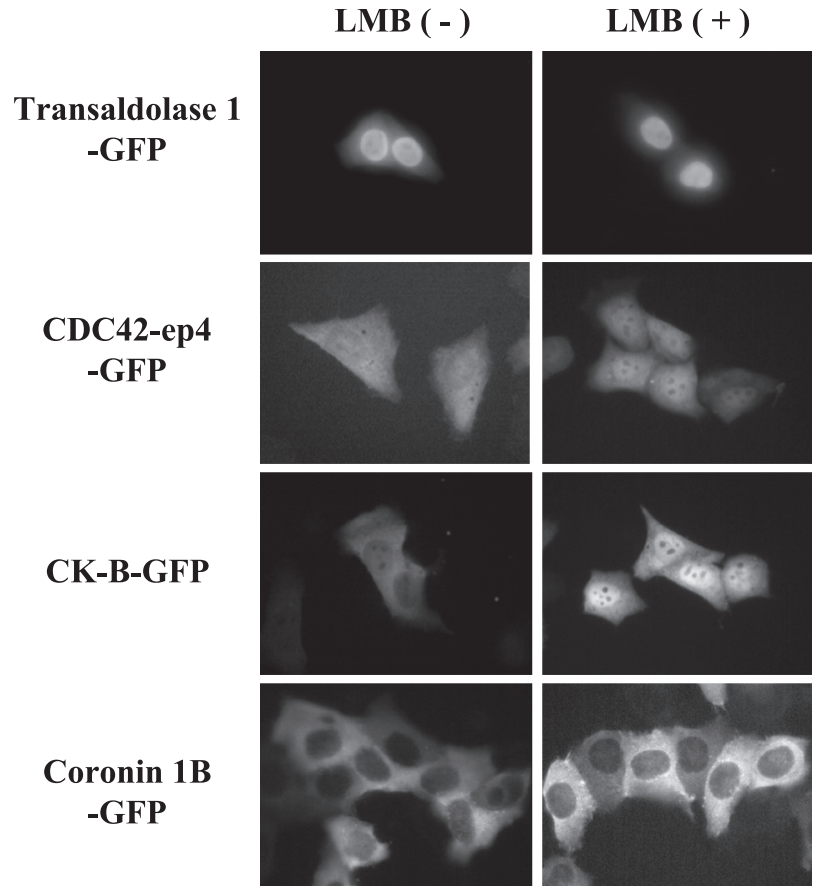

Fig. 6. The effects of LMB on subcellular protein localization. After HeLa cells expressing the GFP-tagged proteins were treated for $2 \mathrm{~h}$ with LMB (10 ng/ml), transaldolase-1, CDC42-ep4, and CK-B shifted from the cytoplasm to primarily the nucleus. In contrast, Coronin $1 \mathrm{~B}$ showed a cytoplasmic localization profile. imported into the nucleus by importin $\alpha 5 / \beta$ in vitro, our data indicate that CK-B is localized in the nucleus. Because the CK-B plays a central role in energy transduction and fluctuating energy demands, our results suggest that nuclear transport with importin $\alpha / \beta$ may play a role in the nuclear energy supply.

Other importin $\alpha 5$-interacting proteins include membrane receptor molecules, such as glutamate receptors. After cleavage, the intracellular domain of Notch1 becomes an active transcription factor in the nuclei of neuronal cells, demonstrating that transmembrane proteins can play important roles in intracellular signal transduction (Chan and Jan, 1998; De Strooper et al., 1999). In response to extracellular stimuli, importin $\alpha 5$ may directly bind to the cytoplasmic domains of the identified transmembrane proteins after cleavage or to cytoplasmic anchoring proteins to transduce signals into the nucleus. Alternatively, these membrane receptors may be regulated through unknown cytoplasmic functions of importin $\alpha 5$.

Our proteomic analysis identified many components of postsynaptic densities (PSDs), such as calcium/calmodulindependent protein kinase II (CaMKII), TRIM (tripartite motif-containing) proteins (Trim 3 and Trim 2) and cytoskeletal proteins (MAP2, actin, tubulin), among the importin $\alpha 5$-binding proteins. PSDs of central excitatory synapses play key roles in postsynaptic signal transduction. Previously reported proteomic analyses of PSDs (Walikonis et al., 2000; Jordan et al., 2004; Li et al., 2004; Yoshimura et al., 2004; Peng et al., 2004; Dosemeci et al., 2007) showed
A

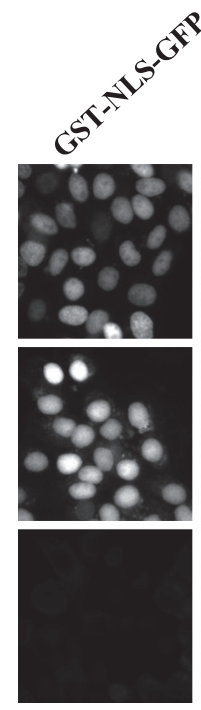

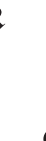<smiles>C1CCCCC1</smiles><smiles>[As]=[As]</smiles>
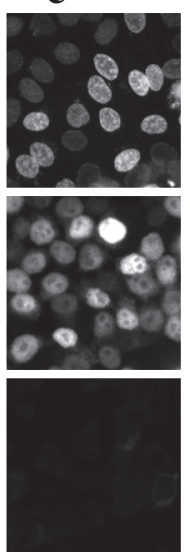
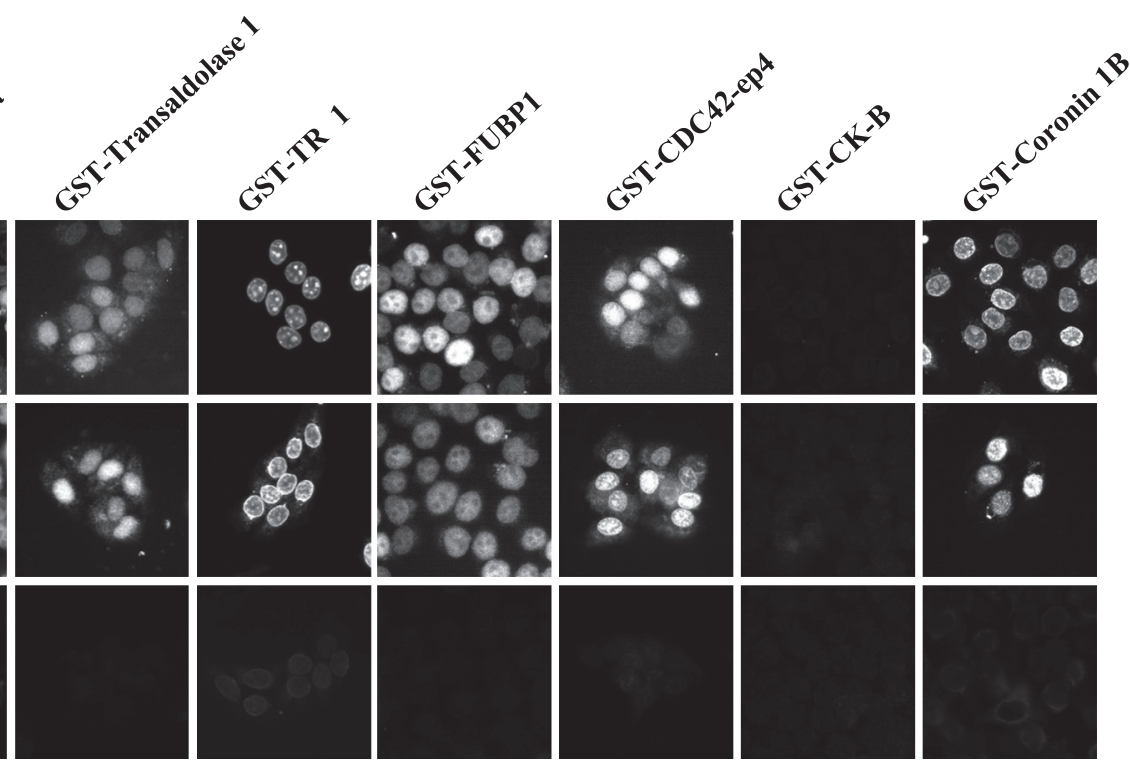

Fig. 7. An in vitro transport assay showed that several of the identified proteins migrated into the nucleus in an importin $\alpha 5 / \beta$-dependent manner. Digitonin-permeabilized HeLa cells were incubated with GST-NLS-GFP or GST fusion proteins. A, Experiments were performed in the presence of importin $\alpha 5$, importin $\beta$, RanGDP, p10/NTF2, and an ATP regeneration system for $20 \mathrm{~min}$ at $30^{\circ} \mathrm{C}$. B, Experiments were performed in the presence of cytosolic extracts prepared from mouse Ehrlich ascites tumor cells for $20 \mathrm{~min}$ at $30^{\circ} \mathrm{C}$. C, Experiments were performed in the presence of an ATP regeneration system for $20 \mathrm{~min}$ at $30^{\circ} \mathrm{C}$. GST fusion proteins were detected using anti-GST antibody. 
high concentrations of glutamate receptors and associated proteins, such as PSD-95; signaling complexes (e.g. CaMKII); and cytoskeletal proteins (MAP2, actin, tubulin) together with their associated proteins (MAP kinase, creatine kinase, Hsp 70). As a major constituent of the PSD complex (Dosemeci et al., 2007), the amount of CaMKII in the PSD is regulated by the level of synaptic activity (Dosemeci et al., 2000). Calcium signaling plays a central regulatory role in activity-dependent neuronal gene expression. Trim 3 binds to $\alpha$-actinin 4 (El-Husseini et al., 2000) and may contribute to nervous system development (El-Husseini and Vincent, 1999). Actin is known to shuttle between the nucleus and cytoplasm, and has been implicated in transcriptional regulation (Dingová et al., 2008). Jordan et al. reported that the PSD complex includes several proteins with NLS sequences (Jordan et al., 2004). Although we did not determine whether importin $\alpha 5$ is actually localized in the PSD, we hypothesized that importin $\alpha 5$ may regulate synaptic function and plasticity by transporting molecules from the PSD to the nucleus or through unidentified cytoplasmic functions of importin $\alpha 5$, including interactions with cytoskeletal proteins and/or cytoskeleton-associated proteins. Further studies are required to elucidate the role of importin $\alpha 5$ in synapses.

A variety of transport factors, including importins and exportins, have been identified and general mechanisms governing the transport of proteins into and out of the nucleus have been clarified. Little is known, however, regarding which molecules are carried by each transport factor and when the various molecules are transported into the nucleus. Although it is currently unclear whether all of the proteins in our proteomic data set enter the nucleus, our strategy can be used with tissue samples or cells obtained at different developmental stages to help clarify the spatiotemporal regulation of nucleocytoplasmic signaling under various physiologic conditions.

Acknowledgments. We wish to thank Dr. T. Tachibana and members of our laboratory for valuable discussions. This work was supported in part by a Grant-in-Aid from the Ministry of Education, Culture, Sports, Science and Technology of Japan; the Takeda S1cience Foundation; and JST, CREST.

\section{References}

Adam, S.A., Marr, R.S., and Gerace, L. 1990. Nuclear protein import in permeabilized mammalian cells requires soluble cytoplasmic factors. $J$. Cell Biol., 111: 807-816.

Balasubramani, M., Day, B.W., Schoen, R.E., and Getzenberg, R.H. 2006. Altered expression and localization of creatine kinase $\mathrm{B}$, heterogeneous nuclear ribonucleoprotein $\mathrm{F}$, and high mobility group box 1 protein in the nuclear matrix associated with colon cancer. Cancer Res., 66: $763-$ 769 .

Baumann, C.T., Maruvada, P., Hager G.L., and Yen, P.M. 2001. Nuclear cytoplasmic shuttling by thyroid hormone receptors. Multiple protein interactions are required for nuclear retention. J. Biol. Chem., 276: $11237-11245$

Cai, L., Marshall, T.W., Uetrecht, A.C., Schafer, D.A., and Bear, J.E. 2007.
Coronin 1B coordinates Arp2/3 complex and cofilin activities at the leading edge. Cell, 128: 915-929.

Chan, Y.M. and Jan, Y.N. 1998. Roles for proteolysis and trafficking in notch maturation and signal transduction. Cell, 94: 423-426.

Chen, L., Roberts, R., and Friedman, D.L. 1995. Expression of brain-type creatine kinase and ubiquitous mitochondrial creatine kinase in the fetal rat brain: evidence for a nuclear energy shuttle. J. Comp. Neurol., 363 : 389-401.

Davis, L.I. 1995. The nuclear pore complex. Annu. Rev. Biochem., 64: 865-896.

De Strooper, B., Annaert, W., Cupers, P., Saftig, P., Craessaerts, K., Mumm, J.S., Schroeter, E.H., Schrijvers, V., Wolfe, M.S., Ray, W.J., Goate, A., and Kopan, R. 1999. A presenilin-1-dependent gammasecretase-like protease mediates release of Notch intracellular domain. Nature, 398: 518-522.

Di, Giovanni S., De, Biase A., Yakovlev, A., Finn, T., Beers, J., Hoffman, E.P., and Faden, A.I. 2005. In vivo and in vitro characterization of novel neuronal plasticity factors identified following spinal cord injury. $J$. Biol. Chem., 280: 2084-2091.

Dingová, H., Fukalová, J., Maninová, M., Philimonenko, V.V., and Hozák, P. 2008. Ultrastructural localization of actin and actin-binding proteins in the nucleus. Histochem. Cell Biol., 131: 425-434.

Dosemeci, A., Reese, T.S., Petersen, J., and Tao-Cheng, J.H. 2000. A novel particulate form of $\mathrm{Ca}(2+) /$ calmodulin-dependent [correction of $\mathrm{Ca}(2+) / C a M K I I-d e p e n d e n t]$ protein kinase II in neurons. J. Neurosci., 20: $3076-3084$

Dosemeci, A., Makusky, A.J., Jankowska-Stephens, E., Yang, X., Slotta, D.J., and Markey, S.P. 2007. Composition of the synaptic PSD-95 complex. Mol. Cell. Proteomics, 6: 1749-1760.

Duncan, R., Bazar, L., Michelotti, G., Tomonaga, T., Krutzsch, H., Avigan, M., and Levens, D. 1994. A sequence-specific, single-strand binding protein activates the far upstream element of c-myc and defines a new DNA-binding motif. Genes Dev., 8: 465-480.

El-Husseini, A.E. and Vincent, S.R. 1999. Cloning and characterization of a novel RING finger protein that interacts with class V myosins. J. Biol. Chem., 274: 19771-19777.

El-Husseini, A.E., Kwasnicka, D., Yamada, T., Hirohashi, S., and Vincent, S.R. 2000. BERP, a novel ring finger protein, binds to alpha-actinin-4. Biochem. Biophys. Res. Commun., 267: 906-911.

Enslen, H., Sun, P., Brickey, D., Soderling, S.H., Klamo, E., and Soderling, T.R. 1994. Characterization of $\mathrm{Ca} 2+/$ calmodulin-dependent protein kinase IV. Role in transcriptional regulation. J. Biol. Chem., 269: 15520-15527.

Görlich, D., Henklein, P., Laskey, R.A., and Hartmann, E. 1996. A 41 amino acid motif in importin-alpha confers binding to importin-beta and hence transit into the nucleus. EMBO J., 15: 1810-1817.

Görlich, D. and Mattaj, I.W. 1996. Nucleocytoplasmic transport. Science, 271: $1513-1518$.

Görlich, D. 1998. Transport into and out of the cell nucleus. EMBO J., 16: 2721-2727.

Görlich, D. and Kutay, U. 1999. Transport between the cell nucleus and the cytoplasm. Annu. Rev. Cell Dev. Biol., 15: 607-660.

Hanz, S., Perlson, E., Willis, D., Zheng, J.Q., Massarwa, R., Huerta J.J., Koltzenburg, M., Kohler, M., van-Minnen, J., Twiss, J.L., and Fainzilber, M. 2003. Axoplasmic importins enable retrograde injury signaling in lesioned nerve. Neuron, 40: 1095-1104.

Henderson, B.R. and Percipalle, P. 1997. Interactions between HIV Rev and nuclear import and export factors: the Rev nuclear localisation signal mediates specific binding to human importin-beta. J. Mol. Biol., 274: 693-707.

Imamoto, N., Tachibana, T., Matsubae, M., and Yoneda, Y. 1995. A karyophilic protein forms a stable complex with cytoplasmic components 
prior to nuclear pore binding. J. Biol. Chem., 270: 8559-8565.

Joberty, G., Perlungher, R.R., and Macara, I.G. 1999. The Borgs, a new family of Cdc42 and TC10 GTPase-interacting proteins. Mol. Cell. Biol., 10: $6585-6597$.

Jordan, B.A., Fernholz, B.D., Boussac, M., Xu, C., Grigorean, G., Ziff, E.B., and Neubert, T.A. 2004. Identification and verification of novel rodent postsynaptic density proteins. Mol. Cell. Proteomics, 3: 857-871.

Köhler, M., Ansieau, S., Prehn, S., Leutz, A., Haller, H., and Hartmann, E. 1997. Cloning of two novel human importin-alpha subunits and analysis of the expression pattern of the importin-alpha protein family. FEBS Lett., 417: 104-108.

Kotera, I., Sekimoto, T., Miyamoto, Y., Saiwaki, T., Nagoshi, E., Sakagami, H., Kondo, H., and Yoneda, Y. 2005. Importin alpha transports CaMKIV to the nucleus without utilizing importin beta. EMBO J., 24: 942-951.

Kurisaki, A., Kose, S., Yoneda, Y., Heldin, C.H., and Moustakas, A. 2001. Transforming growth factor-beta induces nuclear import of Smad3 in an importin-betal and Ran-dependent manner. Mol. Biol. Cell, 12: 10791091.

Lam, M.H., Briggs, L.J., Hu, W., Martin, T.J., Gillespie, M.T., and Jans, D.A. 1999. Importin beta recognizes parathyroid hormone-related protein with high affinity and mediates its nuclear import in the absence of importin alpha. J. Biol. Chem., 274: 7391-7398.

Lee, S.J., Sekimoto, T., Yamashita, E., Nagoshi, E., Nakagawa, A., Imamoto, N., Yoshimura, M., Sakai, H., Chong K.T., Tsukihara, T., and Yoneda, Y. 2003. The structure of importin-beta bound to SREBP-2: nuclear import of a transcription factor. Science, 302: 1571-1575.

Li, K.W., Hornshaw, M.P., Van Der Schors, R.C., Watson, R., Tate, S., Casetta, B., Jimenez, C.R., Gouwenberg, Y., Gundelfinger, E.D., Smalla, K.H., and Smit, A.B. 2004. Implications of the diverse protein functional groups for the integration of synaptic physiology. J. Biol. Chem., 279: 987-1002.

Mattaj, I.W. and Englmeier, L. 1998. Nucleocytoplasmic transport: the soluble phase. Annu. Rev. Biochem., 67: 265-306.

Matthews, R.P., Guthrie, C.R., Wailes, L.M., Zhao, X., Means, A.R., and McKnight, G.S. 1994. Calcium/calmodulin-dependent protein kinase types II and IV differentially regulate CREB-dependent gene expression. Mol. Cell. Biol., 14: 6107-6116.

Miki, T., Okawa, K., Sekimoto, T., Yoneda, Y., Watanabe, S., Ishizaki, T., and Narumiya, S. 2009. mDia2 shuttles between the nucleus and the cytoplasm through the importin-\{alpha $/\{$ beta $\}$ - and CRM1-mediated nuclear transport mechanism. J. Biol. Chem., 284: 5753-5762.

Miyamoto, Y., Hieda, M., Harreman, M.T., Fukumoto, M., Saiwaki, T., Hodel, A.E., Corbett, A.H., and Yoneda, Y. 2002. Importin alpha can migrate into the nucleus in an importin beta- and Ran-independent manner. EMBO J., 21: 5833-5842.

Nagoshi, E., Imamoto, N., Sato, R., and Yoneda, Y. 1999. Nuclear import of sterol regulatory element-binding protein-2, a basic helix-loop-helixleucine zipper (bHLH-Zip)-containing transcription factor, occurs through the direct interaction of importin beta with HLH-Zip. Mol. Biol. Cell, 10: 2221-2233.

Nishi, K., Yoshida, M., Fujiwara, D., Nishikawa, M., Horinouchi, S., and
Beppu, T. 1994. Leptomycin B targets a regulatory cascade of crm1, a fission yeast nuclear protein, involved in control of higher order chromosome structure and gene expression. J. Biol. Chem., 269: 6320-6324.

Peng, J., Kim, M.J., Cheng, D., Duong, D.M., Gygi, S.P., and Sheng, M. 2004. Semiquantitative proteomic analysis of rat forebrain postsynaptic density fractions by mass spectrometry. J. Biol. Chem., 279: 2100321011.

Pollard, V.W., Michael, W.M., Nakielny, S., Siomi, M.C., Wang, F., and Dreyfuss, G. 1996. A novel receptor-mediated nuclear protein import pathway. Cell, 86: 985-994.

Sechi, S. and Chait, B.T. 1998. A tool in peptide mapping and protein identification. Anal Chem., 70: 5150-5158.

Sekimoto, T., Imamoto, N., Nakajima, K., Hirano, T., and Yoneda, Y. 1997. Extracellular signal-dependent nuclear import of Stat1 is mediated by nuclear pore-targeting complex formation with NPI-1, but not Rch1. EMBO J., 16: 7067-7077.

Seo, S.B., Macfarlan, T., McNamara, P., Hong, R., Mukai, Y., Heo, S., and Chakravarti, D. 2002. Regulation of histone acetylation and transcription by nuclear protein pp32, a subunit of the INHAT complex. $J$. Biol. Chem., 277: 14005-14010.

Shevchenko, A., Wilm, M., Vorm, O., and Mann, M. 1996. Mass spectrometric sequencing of proteins silver-stained polyacrylamide gels. Anal. Chem., 68: 850-858.

Stewart, M., Baker, R.P., Bayliss, R., Clayton, L., Grant R.P., Littlewood, T., and Matsuura, Y. 2001. Molecular mechanism of translocation through nuclear pore complexes during nuclear protein import. FEBS Lett., 498: 145-149.

Tsuji, L., Takumi, T., Imamoto, N., and Yoneda, Y. 1997. Identification of novel homologues of mouse importin alpha, the alpha subunit of the nuclear pore-targeting complex, and their tissue-specific expression. FEBS Lett., 416: 30-34.

Walikonis, R.S., Jensen, O.N., Mann, M., Provance, D.W.J., Mercer, J.A., and Kennedy, M.B. 2000. Identification of proteins in the postsynaptic density fraction by mass spectrometry. J. Neurosci., 20: 4069-4080.

Wozniak, R.W., Rout, M.P., and Aitchison, J.D. 1998. Karyopherins and kissing cousins. Trends Cell Biol., 8: 184-188.

Yasuhara, N., Shibazaki, N., Tanaka, S., Nagai, M., Kamikawa, Y., Oe, S., Asally, M., Kamachi, Y., Kondoh, H., and Yoneda, Y. 2007. Triggering neural differentiation of ES cells by subtype switching of importinalpha. Nat. Cell Biol., 9: 72-79.

Yoneda, Y. 2000. Nucleocytoplasmic protein traffic and its significance to cell function. Genes Cells, 5: 777-787.

Yoshimura, Y., Yamauchi, Y., Shinkawa, T., Taoka, M., Donai, H., Takahashi, N., Isobe, T., and Yamauchi, T. 2004. Molecular constituents of the postsynaptic density fraction revealed by proteomic analysis using multidimensional liquid chromatography-tandem mass spectrometry. $J$. Neurochem., 88: 759-768.

(Received for publication, December 6, 2010, accepted, January 26, 2011 and published online, February 5, 2011) 